\title{
Utilizing the Meaning Response:
}

\section{Naturopathy as Meaning-Based Medicine in}

\author{
the Western Cultural Context
}

\author{
Kayla Marie Ruschkowski*
}

\begin{abstract}
The 'placebo effect' as identified in the Western cultural context is more accurately identified as the 'meaning response.' The meaning response is the unique, human ability to heal through participation in a culturally meaningful healing process. The meaning response makes physiological impacts in the process of meaningful healing, even in cases when the healing paradigm involves biological specificity. Therefore, initiating a meaning response within all healing systems is valuable, even in the Western context, where scientific validation is privileged over meaning-based medicine. In the Western context, meaning-based medicine must appeal to cultural values involving scientific validity and contemporary health concerns. This essay will discuss naturopathy as a culturally relevant, meaning-based medical paradigm within Western culture.
\end{abstract}

Keywords: healing, meaning-based medicine, placebo effect, naturopathy

The placebo effect is a powerful phenomenon that has little to do with the placebo itself and much to do with meaning that is created in a healing context. Because of this, the phenomenon known as the 'placebo effect' is better conceptualized as the 'meaning response.' Meaning is present in all aspects of human experience, and makes physiological impacts in human health and healing. The meaning response in healing is a uniquely human system that is effective, accessible, and culturally specific. Due to globalization, healing systems coexist and overlap, with many people having the opportunity to access both meaning-based medicine ${ }^{1}$ and allopathic medicine. By accessing allopathic medicine where appropriate, alongside

\footnotetext{
${ }^{1}$ In this sentence and hereafter, I use the term 'meaning-based medicine' to refer to healing systems in which biological specificity is not utilized as the primary means of treatment. Biological specificity involves using specific (often pharmaceutical) treatments towards specific ailments in order to derive specific results. This is not to say that meaning-based healing systems do not produce real physiological effects. Rather, these effects are holistic in nature and difficult to measure according to scientific methods. Additionally, their effects are variable and dependent on the meaning system in which they originate.
} 
holistic meaning-based methods, individuals can utilize the benefits of scientific advancement while maximizing the body's natural ability to heal through positive meaning.

In the Western multi-cultural context, individuals have the opportunity to access holistic healing systems originating from various cultural backgrounds. However, individuals are most likely to derive the most therapeutic benefit from healing systems which coincide with their cultural backgrounds (Moerman 2000). Individuals effectively respond to meaningful healing that corresponds with their cultural identification and cultural conceptualization of the body. Thus, individuals of Chinese cultural identification and Indian cultural identification should most positively experience Chinese medicine and Ayurvedic medicine, respectively. Traditional healing systems have arisen from the knowledge systems within their respective cultures, thus legitimating their authenticity and breadth of meaning for individuals within those cultures. However, due to the prominence of scientific specificity in Western medical discourse, options for holistic, meaning-based medicine are more limited for individuals who hold a primarily Western cultural identification. Additionally, scientific discourse has effectively delegitimized non-specific healing methods, treating meaning-based healing as inferior and deceptive (Thompson et al. 2008). Consequently, meaning-based medicine must arise within or alongside the knowledge systems associated with allopathic medicine in order for the majority of the Euro-North American population to access it. In order for meaning-based medicine to be perceived as legitimate, it must be integrated with existing Western paradigms.

Physicians' and patients' rising dissatisfaction with contemporary allopathic medicine stems from the increasing corporatization of medical institutions and the standardization of patient-physician interactions (Donald 2011; Lewis 2003). In this context, individuals are increasingly looking for alternatives (Wardle and Oberg 2011). This essay discusses naturopathic medicine as a healing system which can fill an important niche: the provision of culturally relevant, meaning-based medicine within the Western context. According to the Canadian Association of Naturopathic Doctors (2015), naturopathic medicine is "a distinct primary health care system that blends modern scientific knowledge with traditional and natural forms of medicine." Its origins are attributed to Dr. Benedict Lust, a German who founded the first school of naturopathic medicine in New York in 1901 (Kirchfield and Boyle 1994). Thus, naturopathic medicine derives cultural significance through its origin in the Western context. Today, naturopathic medicine integrates various components of contemporary medical discourse, including an emphasis on evidence-based practices, professional regulation, and scientific validation (Wardle and Oberg
2011). Though many naturopathic practices involve traditional, time-tested techniques, these are blended with emphases of contemporary importance. In this sense, naturopathic medicine is a bridge between the traditional and the contemporary.

This essay includes a discussion of the meaning response, which has been conceptually distorted by its association with inert placebos, as used in drug efficacy trials. In fact, what we observe as the placebo effect is the powerful human capacity to heal through positive meaning during the healing process. Through my discussion of the meaning response, I conclude that our best practice is to maximize the meaning response in medicine, whether or not an allopathic treatment is being applied. In maximizing the meaning response, individuals are empowered to choose and engage in healing processes in which they find meaning. For many people searching for an alternative to allopathy, naturopathy provides a culturally appropriate, meaning-based healing system. It employs a variety of methods to maximize the meaning response, including positive expectancy, patience in the healing process, and an emphasis on health rather than disease (Wardle and Oberg 2011). In this way, naturopathy provides a culturally relevant healing system, utilizing medicine's traditional, meaning-based approach within the knowledge systems of the contemporary Western world.

\section{The Meaning Response}

It has been claimed that the history of medicine could be considered the history of the placebo effect (Benson and Friedman 1996). Prior to the mid-180o's, biological specificity was not conceptualized as a necessary component in the process of healing. However, during the late eighteenth and into the early nineteenth century, the introduction of germ theory and further scientific advancement changed medical practice to involve increased biological specificity (Benson and Friedman 1996). This shift prompted physicians to utilize the specific effects of pharmacologic and surgical interventions towards specific ailments. In the advancement of pharmacology, drug efficacy investigations aimed to control for nonspecific effects. It was in this context that the placebo effect was increasingly recognized. It became necessary to screen out the placebo effect, thus characterizing it as a problem to be eliminated (Benson and Friedman 1996). In this context, "the use of the term 'placebo' communicates faith in the underlying biomedical paradigm - that 'true' healing of real diseases only occurs in the context of a powerful external (chemical or mechanical) intervention, and indicates surprise when this paradigm is challenged" (Thompson et al. 2008:119). For medical practitioners and patients alike, 
the term 'placebo' came to be associated with falsity and deception.

Attempts to define and understand the placebo effect have resulted in confusion and debate (Price et al. 2008), much of which has to do with the paradox inherent in the conceptualization of the placebo effect itself. As defined by drug efficacy trials, a placebo is an inert substance incapable of producing specific effects. Yet, paradoxically, a placebo by definition is something that produces measurable effects. These results are not due to the placebo itself, but to the meaning inherent in a therapeutic context. Moreover, it has been found that the placebo effect is a broader phenomenon than the administration of a 'fake' pill. The demeanor of the physician, the healing atmosphere, and expectations surrounding treatment all have demonstrated healing effects (Moerman and Jonas 2002; Jubb and Bensing 2013; Enserink 1999). Therefore, the placebo effect is a phenomenon encompassing the holistic process of healing: the patient's psychosocial context and its effects on the patient's experience, brain, and body. These effects are present in all instances of sickness and healing, regardless of whether a placebo is being applied.

Because of its association with an inert pill described as a placebo, "placebo effect" is an inadequate term to identify this dynamic process. Other terms have been proposed in order to convey the complexity of healing that occurs outside of the scientifically specific realm. "Relaxation response" and "remembered healing" are two examples of these terms (Benson and Friedman 1996). However, from an anthropological perspective, this phenomenon may be best labeled as the meaning response (Moerman 2000). Individuals respond to meaning in every facet of experience, and remarkably so when they engage meaningfully in a healing context. This type of meaning response can be defined as "the desirable psychological and physiological effects of meaning in the treatment of illness" (Moerman 2000:52). Conceptualizing the phenomenon in this way transforms how it is addressed. Placebos are understood as inert and to be avoided. Meaning, however, is a powerful and all-encompassing part of human experience. Meaning responses are phenomenally abstract, yet identical only in the sense that they occur in all individuals, in all healing contexts, and throughout time.

Culture consists of shared, interconnected meaning systems. Thus, as much as culture is abstract, interconnected, and variable, so are meaning responses. According to Moerman and Jonas (2002), "insofar as 1) meaning has biological consequence and 2) meanings vary across cultures, we can anticipate that biology will differ in different places, not because of genetics but because of these entangled ideas"(474). Margaret Lock (2001) describes this physiological process as producing "local biologies"(70-71). Variations in placebo response support the existence of local biologies, with placebo response found to be relatively high in Germany but low in Brazil (Moerman 2000). A striking example of a culturally specific meaning response can be drawn from Chinese culture. Within Chinese medicine and astrology, certain birth years are associated with susceptibility to illnesses involving lumps, nodules, or tumours (Moerman 2000). A study involving 28,169 Chinese Americans and nearly half a million Caucasian Americans found that Chinese Americans die $1.3-4.9$ years earlier if they have a birth year that is considered ill-fated within Chinese culture (Moerman 2000). There was no difference in life expectancy observed within the Caucasian control group. Furthermore, the extent to which this meaning response was observed was correlated with the intensity of the individuals' identification with traditional Chinese culture (Moerman 2000). Pharmaceutical therapy can target the lumps, nodules, and tumours; however, a culturally specific meaning response works on the abstract level, where the idea of ill fate exists.

The way that meaning is physiologically experienced depends in part on one's conceptualization of one's own body. According to Mary Douglas (1970), the body adopts and expresses social pressures in manifold ways, which correlate with the cultural categories in which it is perceived. For example, in Orthodox Indian yoga philosophy, the body is a physical trap, which the yoga practitioner aims to escape through his or her ascetic discipline (Olivelle 1995). In this framework, meaningful relief from suffering must occur at a spiritual level which may be in turn experienced physiologically (Olivelle 1995). Many other conceptualizations of the body involve internal channels or energy centers, such as Hatha yoga's chakras and Chinese medicine's internal Qi. These meaning systems provide a conceptual location to which healing is directed. A conceptual location for healing facilitates a meaning response in allopathic medicine, as well. A prominent example is surgery, which is particularly meaningful within the allopathic paradigm for a variety of reasons (Moerman and Jonas 2002). Beyond the shedding of blood, which is inevitably meaningful in itself, "surgical procedures usually have compelling rational explanations, which drug treatments often do not. The logic of arthroscopic surgery is much more sensible and understandable, especially for people in a culture rich in machines and tools, than is the logic of non-steroidal anti-inflammatory drugs" (Moerman and Jonas 2002:473). Whether it is biologically specific or not, a therapeutic meaning response is stimulated through engaging in knowledge of one's body and expecting a healing process to occur.

Utilizing the meaning response towards healing is valuable both on its own and in conjunction with biologically specific therapies. The meaning response is a uniquely human healing system that can be accessed by all individuals. As Benson and Friedman (1996) describe, 
"unlike most other treatments, it is safe and inexpensive and has withstood the test of time" (193). Throughout human history, the meaning response has acted as one of medicine's most potent assets and will continue to do so. Scientific advancement in medicine has opened medical care to include biologically specific methods of healing, which has produced profound benefits. However, in this advancement, the power of the meaning response should not be disregarded or ridiculed. According to Thompson et al. (2008), in doing so we "impoverish the meaning of our medicine to a degree that it simply doesn't work as well as it might any more" (118). By harnessing the meaning response, the role of the health practitioner is expanded and individuals are empowered to engage in all methods of healing.

\section{The Diversity of Truth in Medicine}

Healing is the practice of improving or eliminating the experience of human suffering caused by disease or injury (Gillett 2004). The breadth of approaches to healing is as large as the potential varieties of human suffering. Each healing approach is dependent on its own holistic system of knowledge that legitimates its accepted truths (Gillett 2004). In medicine, as in all aspects of cultural life, "all knowledge claims arise from subjective positions within discourses where power relations legitimate certain claims as being true and their associated methods as being the correct way to arrive at the truth" (Gillett 2004:728). A scientific medical approach is but one of the approaches to arriving at truth. It is notable that the conceptualization of the placebo effect as harmful or deceitful is legitimated in part by institutional discourse that has an economic stake in the biological paradigm. This is not to say that the biological paradigm is harmful or false; rather, it is central to progress in medicine and the reduction of suffering. Nonetheless, it is incorrect to assert the biological paradigm as the only truth, or to use it to conceptualize all potentialities for healing.

In the contemporary world, the institutions and cultural bodies that legitimate the biological medical paradigm hold tremendous power, and so "an atmosphere is created whereby any views that significantly depart from statistical methodologies based in the natural sciences are regarded as inferior or suspect" (Gillett 2004:730). This atmosphere promotes an agenda to disprove methods of healing that occur without biological specificity. These nonspecific healing methods include a variety of culturally complex and natural healing methods. However, regardless of scientific validation, healing occurs in culturally meaningful healing environments. In some cases, scientific specificity enhances treatment; yet, in $60-90 \%$ of patient visits to physicians, surgical procedures and pharmaceutical agents are of limited therapeutic use (Benson and Friendman 1996). Furthermore, in the case that allopathic remedies produce desired results, this in itself does not disprove other methods. Because of the complexity of human experience and suffering, "there may not be any unitary or single coherent body of knowledge that will serve the diverse purposes of medicine" (Gillett 2004:730). Therefore, the most valuable discourse around health and healing in a multi-cultural context involves plurality and diversity.

Just as diversity in healing paradigms offers individuals more opportunity for meaningful healing, flexibility should be used within paradigms to maximize meaning response. Here, the anthropological concept of narrative analysis is useful to conceptualize the fluidity and the constructional process of suffering and healing. According to Cheryl Mattingly (1994), "we make as well as tell stories of our lives and this is of fundamental importance in the clinical world" (811). The experience of illness and suffering often includes a narrative loss as well as a physical loss, "the fracturing of a life story as patients restructure lives in new ways" (Mattingly 1994:814). It is the role of the physician to enter the patient's fractured narrative, invoke a sense of what it means to be healed, and set this story in motion (Mattingly 1994). Here, the patient must be meaningfully connected to a physician, healing paradigm, and healing process that facilitates this process. All healing processes that create this connection with the patient are valuable. Naturopathic medicine is one of these; its attributes and applicability to the Western cultural context are discussed below.

\section{Naturopathy within the Western Context}

According to Moerman (2000), there are three types of healing processes: "autonomous ones based on the immunological or homeostatic processes of the body, specific ones based on the pharmacological or physical dimensions of the healing process, and meaningful ones, based on knowledge and interaction" (56). Naturopathic medicine aims to engage with all three of these healing processes. It is a system of primary health care that represents the practice and philosophy of selecting and applying complex, individualized, multi-modality treatment regimens (Elder 2013). Modalities of treatment include Asian medicine/acupuncture, botanical medicine, clinical nutrition, homeopathic medicine, health psychology, and lifestyle counseling. According to the Canadian Association of Naturopathic Doctors (2015), the naturopathic philosophy is to "stimulate the innate healing power of the body." One of these innate healing systems involves the 
meaning response; thus, naturopathy has explicitly utilized the meaning response since its inception.

The number of people turning to alternative therapies is growing (Wardle and Oberg 2011). In some instances, alternative therapies serve to complement allopathic medicine. In other instances, individuals pursue alternate therapies due to dissatisfaction with the allopathic paradigm. With the cultural transition brought by the prominence of pharmaceuticals and technomedicine, the relationship between physician and patient has changed (Lewis 2003). This has included less patient-physician interaction and more systematized, standardized care (Lewis 2003). Furthermore, increased control of medical institutions by pharmaceutical corporations further fragments the patient-physician relationship. In this context, psychiatrists and nurses alike observe they must treat patients like customers, which robs both parties of their individual complexities (Donald 2011). According to Brad Lewis, who traced the cultural emergence of Prozac, "in this environment, physicians and psychiatrists are in danger of becoming glorified distributors of the new technologies - sort of like new-car dealers with a medical certificate" (2003:122). It is in this context that patients seek the personalized, patient-centered care that naturopathic medicine offers.

The production and transmission of knowledge within Western societies relies heavily on scientific discourse. Naturopathic medicine lies in a unique position because many of its practices derive from traditional knowledge systems, yet these practices are legitimated by structures and discourses that are important within contemporary Western culture. This is due in part to naturopathic medicine's origin within Western culture and efforts to make it accessible to the wider Western population. For example, in Canada, "the naturopathic medical profession's infrastructure includes accredited educational institutions, professional licensing, national standards of practice, participation in many federal health committee initiatives, and a commitment to state-of-theart scientific research" (Canadian Association of Naturopathic Doctors 2015). Naturopathic medicine also incorporates today's collaborative global mindset. It combines therapies sourced from knowledge systems around the world, making them accessible to the Western population through scientific validation.

In addition to having cultural relevancy, naturopathic medicine has proven to be highly adaptable to cultural change. Since the 1970's, there has been increased emphasis placed upon evidence-based practices (EBP) within all health professions (Leach and Gillham 2011). According to a study by Leach and Gillham (2011) that evaluated attitudes and use of EBP by Complementary and Alternative Medicine (CAM) practitioners, CAM practitioners value EBP in their practice. According to this study, these practitioners "valued evidence-based practice, actively use EBP, and reported good or advanced skills in searching and critically appraising literature" (Leach and Gillham 2011:135). Additionally, in contemporary health discourse there has been an emphasis on thinking upstream; that is, managing risk factors for illness onset and appraising the economic value of illness prevention (Homer et al. 2007). Naturopathic medicine's emphasis on illness prevention and education situates the naturopathic paradigm to respond effectively to these demands.

The conceptualization of health is fluid and influenced by cultural change. The World Health Organization (1946) defines health as a "state of complete physical, mental, and social well-being and not merely the absence of disease or infirmity." This concept of health has become more central within Western culture recently, with new discourses defining health as "a resource for everyday life, not the objective of living . . . a positive concept emphasizing social and personal resources as well as physical capacities" (Wardle and Oberg 2011:1079). Naturopathic medicine, with its emphasis on health rather than disease, is culturally situated to respond and adapt to contemporary definitions and concerns around health. For example, obesity is one of the primary health concerns in the Western world. Allopathic medicine currently has limited options for this health concern, with only $35-40 \%$ of visits to physicians including counseling on diet, physical activity, and stress management (Wardle and Oberg 2011). Naturopathic medicine, however, emphasizes health and lifestyle education within almost every clinical encounter (Wardle and Oberg 2011). In this way, naturopathic physicians "provide the patient with opportunities to attribute meaning and attain mastery over skills that lead to empowerment and improved health outcomes" (Thompson et al. 2008:118). Thus, naturopathic medicine's approach is in alignment with current discourses around health and well-being.

The meaning response is a powerful, uniquely human healing system that should be harnessed in all healing systems. However, the prominence of scientific discourse and standardization of care limit access to meaning-based healing in Western allopathic settings. In this context, individuals are seeking alternative and complementary treatments such as naturopathy. Naturopathy is a culturally relevant healing system that bridges traditional meaning-based medicine with contemporary Western culture. It will not replace allopathic methods, but can contribute to the diversity that is to be embraced within medicine. Naturopathy provides a unique healing system, utilizing medicine's traditional meaningbased approach within the knowledge systems of the contemporary Western world. In this way, it offers culturally relevant healing that facilitates a positive meaning response. 


\section{References}

Benson, Herbert, and Richard Friedman 1996 Harnessing the Power of the Placebo Effect and Renaming it 'Remembered Wellness.' Annual Review of Medicine 47:193-199.

Canadian Association of Naturopathic Doctors 2015 What is Naturopathic Medicine? http://www.cand.ca, accessed April 2, 2015.

Donald, Alistair 2011 The Wal-Marting of American Psychiatry: An Ethnography of Psychiatric Practice in the Late 2oth Century. Culture, Medicine and Psychiatry 25(4):427-439.

Douglas, Mary 1978 Natural Symbols: Explorations in Cosmology. London: Barrie and Jenkins.

Elder, Charles R. 2013 Integrating Naturopathy: Can we Move Forward? The Permanente Journal 17(4):80.

Enserink, Martin 1999 Can the Placebo be the Cure? Science 284(5412):238-240.

Gillett, Grant 2004 Clinical Medicine and the Quest for Certainty. Social Science \& Medicine 58(4):727-738.

Homer, Jack, Gary Hirsch, and Bobby Milstein 2007 Chronic Illness in a Complex Health Economy: The Perils and Promises of Downstream and Upstream Reforms. System Dynamics Review 23(3):313-343.

Jubb, Jayne, and Jozien M. Bensing 2013 The sweetest pill to swallow: How Patient Neurobiology can be Harnessed to Maximize Placebo Effects. Neuroscience \& Biobehavioral Reviews 37(10):2709-2720.

Kirchfield, Friedhelm, and Wade Boyle 1994 Nature Doctors: Pioneers in Naturopathic Medicine. Portland OR: Medicina Biologica.
Leach, Matthew, and David Gillham 2011 Are Complementary Medicine Practitioners Implementing Evidence Based Practice? Complementary Therapies in Medicine 19(3):128136.

Lewis, Bradley E. 2003 Prozac and the Post-human Politics of Cyborgs. Journal of Medical Humanities 24(12):29-63.

Lock, Margaret 2001 The Tempering of Medical Anthropology: Troubling Natural Categories. Medical Anthropology Quarterly 15(4):478-492.

Mattingly, Cheryl 1994 The Concept of Therapeutic Emplotment. Social Science \& Medicine 38(5):811-822.

Moerman, Daniel E. 2000 Cultural Variations in the Placebo Effect: Ulcers, Anxiety, and Blood Pressure. Medical Anthropology Quarterly 14 (1):51-72.

Moerman, Daniel E., and Wayne B. Jonas 2002 Deconstructing the placebo effect and finding the meaning response. Annals of Internal Medicine 136(6):471-476.

Olivelle, $\mathrm{P}$.

1995 Deconstruction of the Body in Indian Asceticism. In V. L. Wimbush (Ed.) Asceticism (pp. 188-210). New York, NY: Oxford University Press.

Price, Donald D., Damien G. Finniss, and Fabrizio Benedetti 2008 A Comprehensive Review of the Placebo Effect: Recent Advances and Current Thought. Annual Review of Psychology 59:565-590.

Thompson, Jennifer J., Cheryl Ritenbaugh, and Mark Nichter

2008 Reconsidering the Placebo Response from a Broad Anthropological Perspective. Culture, Medicine, and Psychiatry 33(1):112-152.

Wardle, Jon and Erika B. Oberg 2011 The Intersecting Paradigms of Naturopathic Medicine and Public Health: Opportunities for Naturopathic Medicine. The Journal of Alternative and Contemporary Medicine 17(11):1079-1084. 
World Health Organization

1946 Preamble to the Constitution for the World Health Organization as adopted by the International Health Conference, New York.

Signed on July 1946 and entered into force on 7 April 1948. 
